\title{
Le laboratoire de dosimétrie passive SCPRI-OPRI-IRSN
}

\begin{abstract}
A. BIAU
Ce laboratoire, situé au Vésinet, a été une entité du Service central de protection contre les rayonnements ionisants (SCPRI), puis de l'Office de protection contre les rayonnements ionisants (OPRI) et enfin de l'Institut de radioprotection et de sûreté nucléaire (IRSN), créée pour assurer la surveillance dosimétrique dans tous les domaines d'utilisation des rayonnements ionisants.
\end{abstract}

\subsection{La création du laboratoire}

Le laboratoire a été mis en place à la fin des années 1950 peu de temps après la création du SCPRI en 1957 par Pierre Pellerin sous la tutelle du Ministère de la santé pour contrôler les activités mettant en œuvre des rayonnements ionisants dans des établissements ne dépendant pas du CEA et de la Défense. Cette scission au niveau du CEA a été un gros bouleversement qui n'a pas toujours été bien accepté et a généré des antagonismes qui ont eu la vie dure.

Après un lancement très limité dans des locaux de Fontenay aux Roses, le laboratoire a pris son essor dès son installation dans les installations du Vésinet avec Jean Pierre Moroni. À partir de 1958 ce laboratoire a occupé plusieurs locaux du Vésinet au fil de la progression du nombre de dosimètres à traiter car le traitement manuel des dosimètres photographiques nécessite de la place et de la main d'œuvre.

\subsection{Techniques utilisées}

Jusqu'en 2008 la technique utilisée a été la dosimétrie photographique pour la surveillance réglementaire de base. En complément la dosimétrie des extrémités était assurée par des dosimètres thermo-luminescents et la dosimétrie des neutrons rapides par des émulsions photographiques Kodak NTA puis par des détecteurs solides de traces PN3 à partir de 2000.

\subsubsection{Dosimétrie photographique}

Au départ le dosimètre utilisé était le dosimètre Kodak français de type Chassende-Baroz, dosimètre photographique papier dont la lecture se faisait avec 
des densitomètres à réflexion (voir chapitre 2). Cette technique a été utilisée jusqu'à la fin des années 1960. Lorsque je suis arrivé au SCPRI en 1973, la technique en cours était le dosimètre photographique film de type Kodak type 3 et Agfa, alternativement d'un mois à l'autre.

Ces dosimètres étaient lus avec des densitomètres à transmission et comportaient chacun deux films avec des sensibilités forte et faible. L'inconvénient de ces types de dosimètres venait du volume de développement car pour chaque dosimètre il y avait deux films à développer et le film portant l'émulsion la moins sensible était vierge dans environ $90 \%$ des cas, ce qui occasionnait une grande quantité de films inutiles qui étaient jetés.

Dans les années 1970 est apparu le dosimètre Kodak US type 2 qui présentait l'avantage de n'être constitué que d'un film portant deux émulsions différentes sur les deux faces. Le principal intérêt venait de la diminution d'un facteur deux du volume de développement. En contrepartie il y avait la contrainte, pour les dosimètres irradiés à plus de $4 \mathrm{mSv}$ de « gratter » l'émulsion sensible saturée pour pouvoir lire sur l'émulsion lente mais heureusement cela ne concernait que $10 \%$ des dosimètres environ.

Ce dosimètre sera utilisé à partir de 1975 jusque dans les années 2000.

\subsubsection{Le cycle de traitement du dosimètre photographie}

\subsubsection{Marquage des dosimètres}

Le dosimètre papier comportait une plage auto-marquante qui permettait une numérotation bien visible après développement. Pour les dosimètres films, le marquage était beaucoup plus difficile. La numérotation des dosimètres se faisait sur l'enveloppe papier avec une pression suffisante pour marquer l'émulsion des films à l'intérieur mais pas trop forte pour éviter le risque de perforation de l'enveloppe entrainant une entrée de lumière et la détérioration complète du film (figure 7.1).

La lecture du numéro était délicate et impossible lorsque le film présentait un noircissement significatif. Pour limiter le risque d'erreur d'identification les dosimètres étaient reclassés en ordre numérique à leur retour au laboratoire (figure 7.2). Ainsi chaque mois du $1^{\mathrm{er}}$ au 10 , les dosimètres étaient classés dans des boites de classement de 2000 dosimètres. Vers le 10, lorsque la décision de débuter le traitement des dosimètres était prise, les boites de classement étaient complétées en incluant des plaquettes « fantômes » pour remplacer les dosimètres manquants et reconstituer ainsi la séquence numérique initiale. 


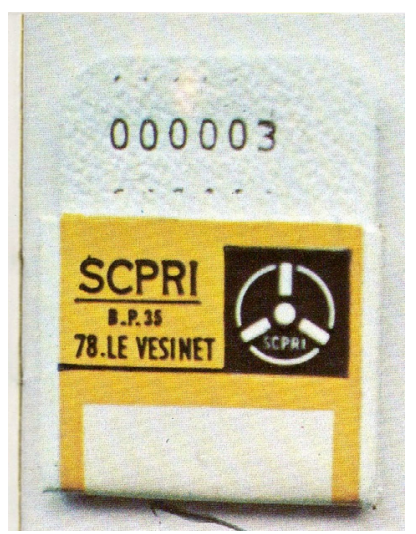

Figure 7.1 - Dosimètre SCPRI (SCPRI, 1979).

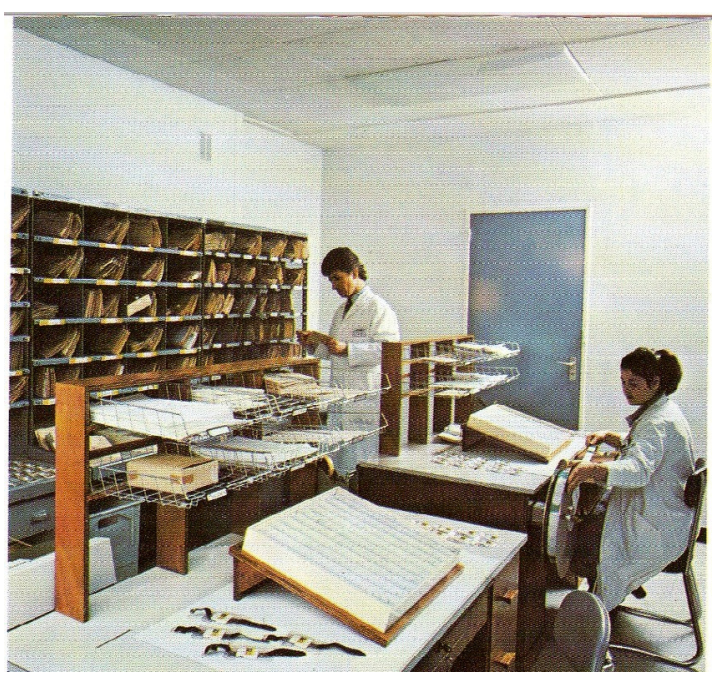

Figure 7.2 - Tri postal et classement des dosimètres retournés au SCPRI pour traitement (SCPRI, 1979).

La plupart des laboratoires ont utilisé le marquage par pression sauf EDF qui disposait d'une machine (robot) numérotant, sous forme de marques codées par exposition aux rayons $\mathrm{X}$ de faible énergie d'une partie du film dosimètre.

Le nombre de dosimètres croissant d'année en année, cette procédure de classement devenait de plus en plus lourde, c'est pourquoi nous avons décidé en 
1995 de la remplacer par une procédure de prise en compte des dosimètres au fur et à mesure de leur arrivée au laboratoire :

L'identification du dosimètre était matérialisée par une étiquette portant le numéro d'abonnement de l'établissement, les nom et prénom du porteur et le $\mathrm{n}^{\circ} \mathrm{du}$ dosimètre en clair et en code barres.

Le début du traitement avait lieu dès le $1^{\mathrm{er}}$ jour du mois au fil de leur retour au laboratoire et les doses anormales pouvaient ainsi être détectées plus rapidement

Dans le laboratoire, sur chaque dosimètre le numéro en code barres était lu sur l'enveloppe et transposé sur une étiquette collée sur le film (figures 7.3 et 7.4).
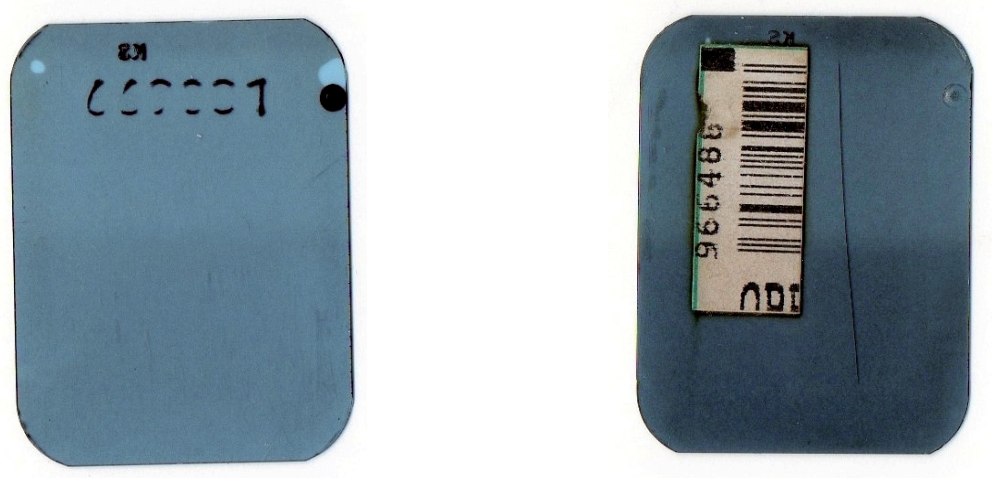

Figure 7.3 - Dosimètre numéroté par pression avant 1995 (à gauche) et par code barres depuis 1995 (à droite).

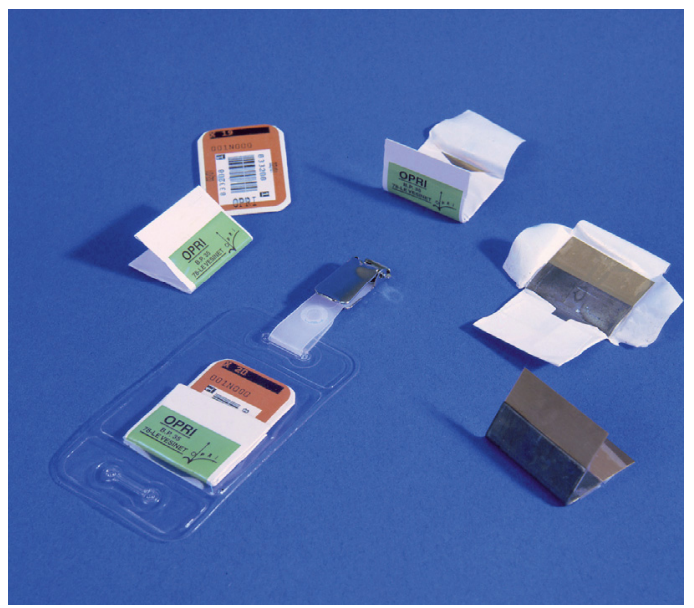

Figure 7.4 - Présentation du dosimètre photographique OPRI- étiquette code barres, étuis porte écrans (cuivre et plomb) et pochette plastique. 


\subsubsection{Condition de port des dosimètres}

Après inscription au laboratoire de dosimétrie du SCPRI-OPRI, le premier mois l'établissement recevait une fiche portant les numéros et noms de tous les abonnés et les dosimètres correspondants et en plus un dosimètre Témoin devant rester à l'abri des rayonnements pour servir de référence durant la période de port. Le premier mois les dosimètres étaient complets sous forme d'un badge comprenant le dosimètre photographique, identifié par un numéro à 6 chiffres, et inséré dans un étui porte écrans contenant un écran de plomb et un écran de cuivre, le tout étant placé dans une pochette plastique munie d'une attache pour fixer le dosimètre sur le vêtement du porteur. À partir du deuxième mois seuls les dosimètres photographiques étaient adressés au responsable de l'établissement abonné qui retournait les dosimètres du mois précédent et insérait les nouveaux dosimètres dans les étuis et pochettes plastiques et les distribuait aux personnels surveillés.

\subsubsection{Développement des dosimètres}

Avant 1995, une fois le début du traitement décidé, les dosimètres étaient collés bout à bout sur un ruban adhésif constituant un long ruban de 40 mètres pour 1000 dosimètres pour éviter toute permutation dans l'obscurité du laboratoire de développement (figure 7.5). Ces rubans étaient transmis au laboratoire où les films étaient extraits un par un des enveloppes et placés dans des paniers comportant 40 logements. Ces paniers étaient positionnés par 8 sur un axe porte panier pour constituer un ensemble de 320 films. En tête de chaque panier était placé un film

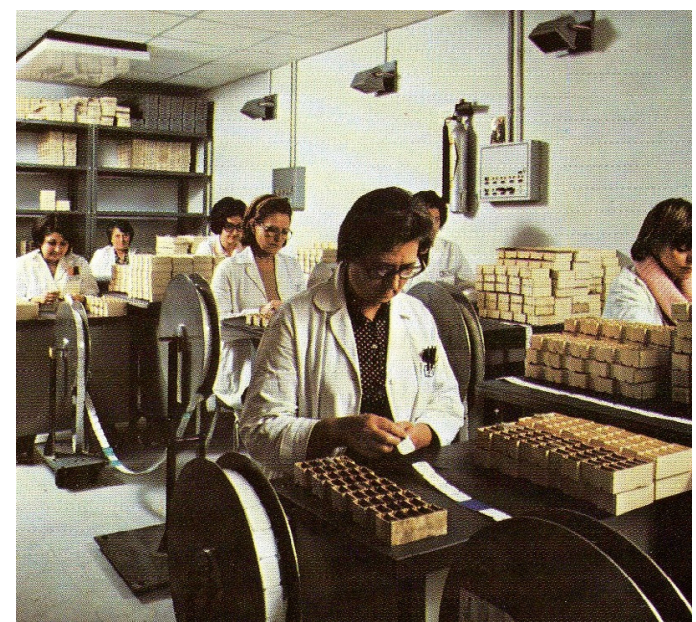

Figure 7.5 - Ouverture des dosimètres SCPRI et mise en panier des films pour développement effectuées sous lumière inactinique (SCPRI, 1979). 
exposé à une source de radium à dose constante et un film vierge pour tenir compte d'éventuelles variations des conditions de développement.

Les films étaient ainsi développés par série de 320 en passant, respectivement, dans les bains de développement, bain d'arrêt et fixateur. Le processus était semiautomatisé et les bains à température régulée.

À partir de 1995, lorsque le système de numérotation a changé, le processus de développement dans les paniers a continué jusqu'à la mise en place d'un système de développement automatisé. Les dosimètres identifiés par un numéro en clair et en codes barres étaient placés dans l'ordre d'arrivée et collés bout à bout pour constituer un grand ruban, la correspondance entre la position du dosimètre et son numéro étant enregistrée.

À partir de 1998 cette opération sera effectuée avec une « rubanneuse » présentée dans la figure 7.6. Ensuite les bobines, ainsi constituées, étaient transférées pour développement dans une machine automatique de développement du type de celles qui sont utilisées dans l'industrie cinématographique.
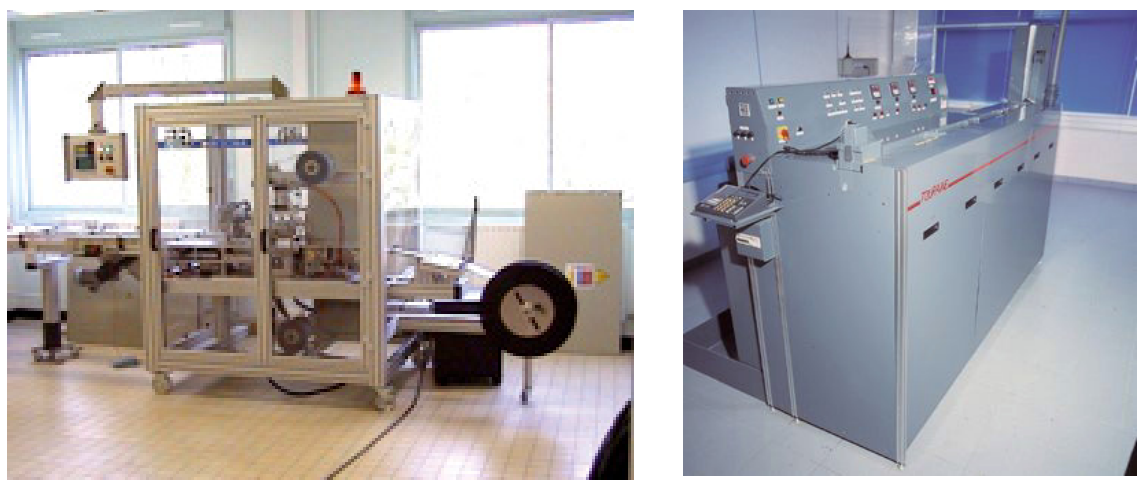

Figure 7.6 - Rubanneuse (à gauche) et développeuse automatiques (à droite) (OPRI).

\subsubsection{Lecture des dosimètres}

À la fin du cycle de développement, après séchage, les dosimètres étaient pris un par un dans les paniers de développement et lus avec un densitomètre en deux points dans la partie sans écran et dans la partie située sous l'écran de plomb (figure 7.7). La partie située sous l'écran de cuivre n'était lue que dans des cas particuliers.

Une fois lu, chaque dosimètre était placé dans un album pour classement et vérification. 


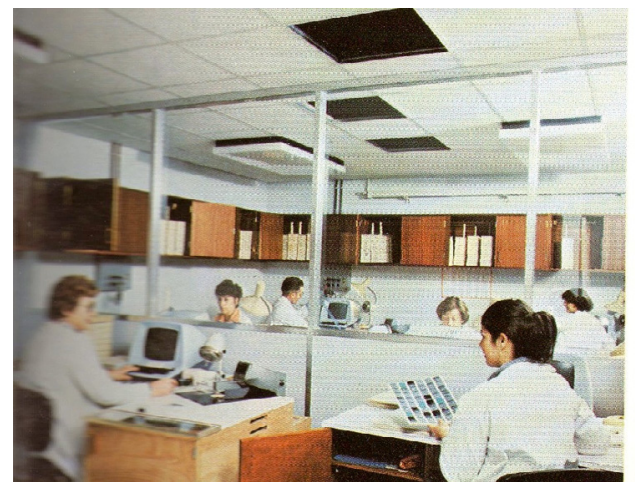

Figure 7.7 - Lecture des dosimètres SCPRI (SCPRI, 1979).

Chaque lecture était enregistrée sur un support informatique (ruban perforé) transmis au service informatique qui éditait un listing portant pour chaque numéro de dosimètre les densités optiques lues et la dose calculée selon un algorithme basé sur des étalonnages mensuels décrits ci-dessous.

Les dosimètres classés dans les albums faisaient ensuite l'objet d'une vérification dite « relecture » en les confrontant avec leur interprétation transcrite sur le listing. Cette relecture portait essentiellement sur les dosimètres ayant reçu une dose supérieure au seuil de mesure et permettait de vérifier la lecture et la conforter ou la modifier en fonction par exemple d'inhomogénéité de densité. Contrairement à ce qui était quelquefois colporté les dosimètres étaient bien lus avec des densitomètres et non à l'œil ou au hasard.

Après la mise en œuvre du système automatique de mise en ruban et de développement, les dosimètres étaient lus un par un en faisant défiler les dosimètres comme le montre la figure 7.8. Les doses étaient calculées au fur à mesure et l'ensemble des résultats transmis au service informatique de Philippe Froissart qui éditait les fiches de résultats destinées au Médecin du travail de chaque établissement abonné.

\subsubsection{5. Étalonnage des dosimètres}

Chaque mois étaient prélevés dans les lots de films destinés aux abonnés, une vingtaine pour effectuer des étalonnages précis car la réponse des films dosimétriques varie selon les émulsions. Ces dosimètres étaient exposés au Bureau international des poids et mesures (BIPM) dans le laboratoire dirigé par le Professeur André Allisy, à des rayons $\mathrm{X}$ de $80 \mathrm{kV}$ et au rayonnement $\gamma$ du cobalt 60 à des doses connues. 


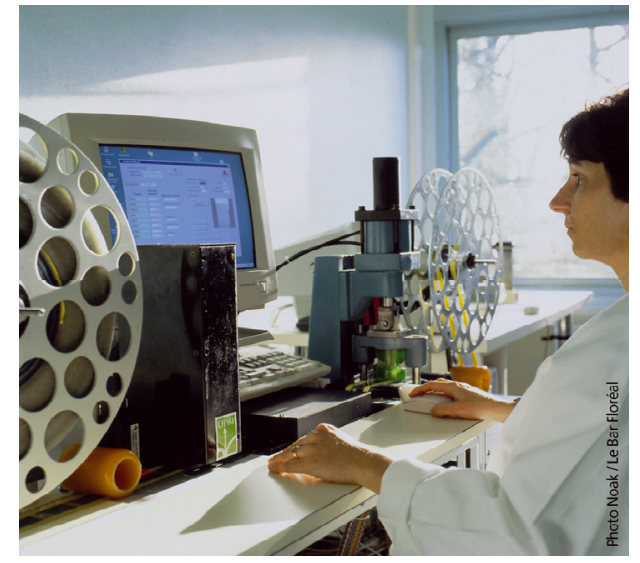

Figure 7.8 - Lecture des dosimètres photographiques OPRI.

À partir des mesures de densité effectuées sur ces dosimètres en fonction des doses les courbes étaient tracées pour déterminer les coefficients de conversion densité-dose selon la formule

$$
\begin{aligned}
\mathrm{D}=\alpha \mathrm{d}^{\mathrm{p}}+\beta \mathrm{d}^{\mathrm{q}} \quad \text { où } \quad \mathrm{D} & =\text { dose } \\
\mathrm{d} & =\text { densité optique }
\end{aligned}
$$

Les quatre courbes concernent l'émulsion sensible partie nue et partie sous l'écran de plomb et l'émulsion lente sous les mêmes plages de mesure. Une fois ces courbes établies et validées les formules correspondantes étaient prises en compte dans le logiciel de calcul des doses.

\subsubsection{Expression des résultats}

Lorsque les dosimètres ont été lus et relus, le service informatique édite les fiches de résultats pour chaque établissement à l'attention du médecin du travail. Sur ces fiches figurent, pour chaque abonné, le numéro du dosimètre, les nom et prénom, l'équivalent de dose, le classement en catégorie I, II ou III et un code d'observation.

Catégorie I : résultat conforme à la réglementation.

Catégorie II : résultat égal ou supérieur à 450 millirems $(4,5 \mathrm{mSv})$ pourrait conduire à un dépassement de limite s'il se reproduisait régulièrement.

Catégorie III : résultat supérieur à 1 rem $(10 \mathrm{mSv})$.

Code d'observation : A : résultat inférieur au seuil de mesure $\mathrm{B}:$ dosimètre non retourné 


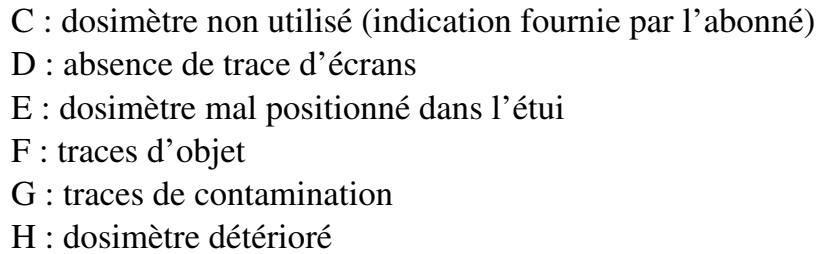

Les résultats classés en catégorie III faisait l'objet d'une lettre jointe à l'envoi des fiches de résultats pour attirer l'attention du médecin du travail. Lorsque le résultat atteignait 25 rems $(250 \mathrm{mSv})$ une lettre plus détaillée était adressée sans attendre l'édition globale des résultats au médecin du travail pour demander une enquête sur les conditions de cette exposition.

À partir de 1986 après la parution du décret du 2 octobre 1986, la présentation des résultats est restée identique mais les lettres au médecin du travail ont changé. Les alertes concernaient les dépassements de limites trimestrielles de $1.5 \mathrm{rem}$ $(15 \mathrm{mSv})$ et annuelles de 5 rems $(50 \mathrm{mSv})$ sur 3 mois et 12 mois glissants.

\subsubsection{Nombre de dosimètres traités annuellement}

Le nombre de dosimètres traités annuellement au laboratoire du Vésinet (SCPRI-OPRI) a progressé de 300000 en 1973 à 1600000 en 2000 pour près de140 000 personnes suivies. La progression a été permanente avec une inflexion dans les années 1990 avec la mise en place du suivi trimestriel pour les abonnés les moins exposés. Cette croissance constante est due au développement permanent des activités professionnelles utilisant des sources de rayonnements ionisants, en médecine d'abord, puis dans les domaines de l'industrie classique et surtout dans le nucléaire civil dans les années 1970. Le nombre d'établissements abonnés a également augmenté dans les mêmes proportions pour atteindre 17000 en 2000. Le tableau I montre la répartition des travailleurs suivis par l'OPRI dans les principaux domaines d'activité professionnelle en 2000.

TABLEAU I

Répartition des abonnés à la surveillance dosimétrique de l'OPRI en 2000 par secteur d'activité (OPRI, 2001).

\begin{tabular}{lcc}
\hline Type d'activité & Nombre de personnes & Pourcentage \\
\hline Médecine & 94265 & $69 \%$ \\
Vétérinaire & 2732 & $2 \%$ \\
Nucléaire- entreprises extérieures & 23225 & $17 \%$ \\
Recherche & 8197 & $6 \%$ \\
Industrie non nucléaire & 2732 & $2 \%$ \\
Divers & 5465 & $4 \%$ \\
Total & 136616 & $100 \%$ \\
\hline
\end{tabular}


Parmi ces abonnés, dans le domaine médical essentiellement, 4921 ont porté, en complément du dosimètre de poitrine, un dosimètre au niveau du poignet, $65 \%$ en radiodiagnostic, $16 \%$ en radiothérapie, $16 \%$ en médecine nucléaire et $0,3 \%$ en radio-immunologie.

\subsubsection{Quelques anecdotes}

Quand on a eu la responsabilité du traitement de plusieurs millions de dosimètres, il y a bien sûr des tas d'histoires anecdotiques ou plus graves à raconter et c'est ce qui fait le charme de ce métier de service public.

Il y a tous les incidents qui ont été révélés ou confirmés par la lecture des dosimètres comme à Tricastin par exemple en juin 2000 où un agent intervenu en zone rouge a reçu une dose qui était sous estimée par le dosimètre opérationnel saturé et dont la valeur réelle a été révélée par le dosimètre photographique et confirmée par la dosimétrie biologique.

Un autre exemple est une exposition significative d'un chirurgien dentiste dont l'appareil de radiologie émettait un rayonnement dans toutes les directions en raison de la défaillance du collimateur. C'est la succession de résultats significatifs qui a révélé cette situation.

D'autres évènements plus ennuyeux se sont produits en raison d'une mauvaise appréciation de l'intérêt de la surveillance réglementaire. L'idée que les dosimètres n'étaient pas fiables ou pas systématiquement interprétés était répandue dans certains milieux et pour s'en assurer il arrivait que les dosimètres soient volontairement exposés pour démontrer l'inefficacité de cette surveillance.

Les personnes (rares heureusement) qui ont eu l'idée d'exposer volontairement des dosimètres n'étaient pas tous des spécialistes en dosimétrie et ont, soit délivré des doses très faibles inférieures au seuil de mesure et dans ce cas le résultat $(0 \mathrm{mSv})$ semblait confirmer leur doute, soit exposés à des doses très élevées entrainant une lettre circonstanciée au médecin du travail (voir plus haut) et une enquête qui a révélé la supercherie. Ce genre de situation a été parfois très mal perçu par l'employeur avec les conséquences que l'on imagine.

Des situations plus fantaisistes ont eu des conséquences moins graves comme on peut l'observer sur la figure 7.9.

L'artiste naturiste était un membre d'un service de médecine du travail effectuant des contrôles radiologiques itinérants (camions radiologiques) (dans ce cas il ne s'agissait pas d'une exposition mais de la substitution dans l'enveloppe du dosimètre du film par une diapositive qui a suivi le cycle de traitement des dosimètres 

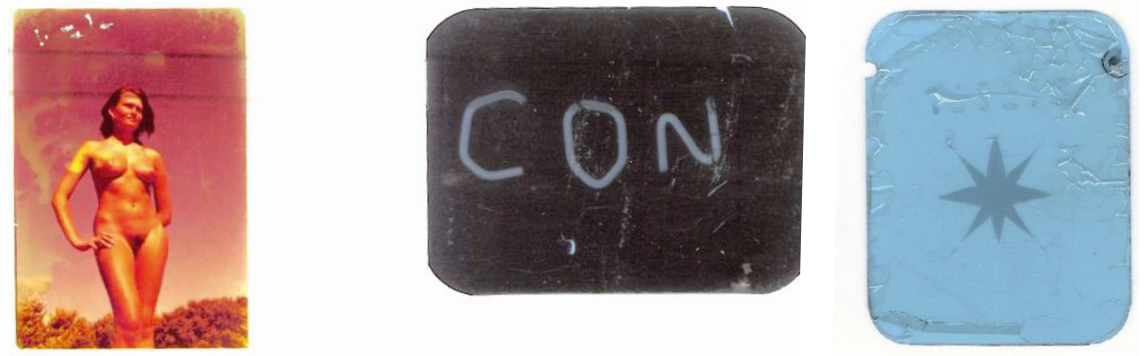

Figure 7.9-Dosimétrie fantaisiste.

sans être altérée). Le grossier personnage qui a confectionné avec des trombones le mot révélé sur le dosimètre était un étudiant. L'étoile a été découpée dans un matériau absorbant (par exemple enveloppe de protection d'une plaque de chocolat) et le dosimètre exposé à des rayons $\mathrm{X}$ de basse énergie.

A contrario, un exemple de sérieux nous a été donné avec le retour d'un dosimètre croqué par un porc (figure 7.10). Ce dosimètre a été retourné avec une lettre relatant avec beaucoup de précisions les circonstances de cet « incident».

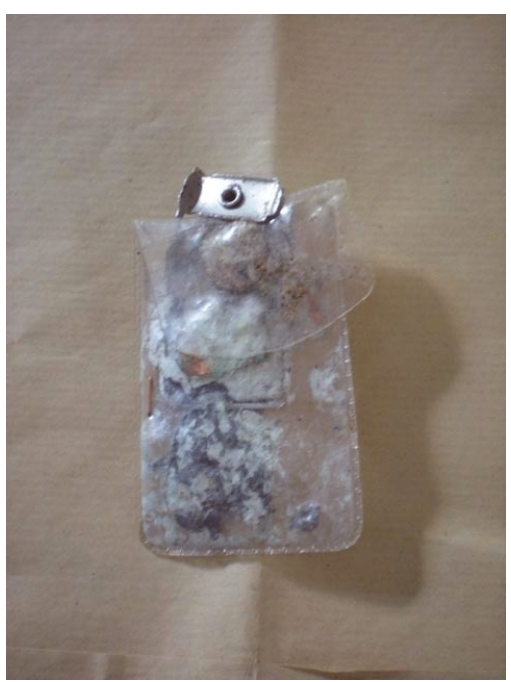

Figure 7.10 - Dosimètre croqué par un porc. 
Enfin un aspect important du travail dans un laboratoire de dosimétrie photographique est le côté humain car la difficulté d'automatisation implique la nécessité d'avoir des effectifs de personnel suffisants, c'est un handicap mais aussi une chance.

Compte tenu des délais contraints en dépit de toute circonstance extérieure pénalisante, grèves postales, incidents sur des matériels de traitement, absences de personnel pour maladie, il fallait procéder à ce que l'on appelait des opérations « coup de poing » pour limiter les retards ou les rattraper. L'esprit d'équipe est fondamental dans ce genre de travail et malgré la charge de travail très lourde il y a eu de très grands moments de plaisir.

\subsubsection{Dosimétrie par thermoluminescence (TLD)}

Dès les années 1970, Pierre Brun a utilisé des dosimètres thermo-luminescents au fluorure de lithium (FLi) et au fluorure de calcium $\left(\mathrm{F}_{2} \mathrm{Ca}\right)$, d'abord sous forme de poudre puis sous forme de pastilles (figure 7.11). Ces dosimètres ne servaient pas à assurer la surveillance dosimétrique des travailleurs mais à réaliser des mesures de dose dans le cadre d'études de poste, des mesures d'environnement et surtout des études dosimétriques sur des patients lors d'applications potentiellement irradiantes. Ainsi une étude originale pour l'époque, a été réalisée dans un hôpital de Lyon sur des nouveaux nés souffrant de malformations cardiaques et faisant l'objet d'examens radiologiques in toto compte tenu de leur petite taille (Amiel et al., 1976).
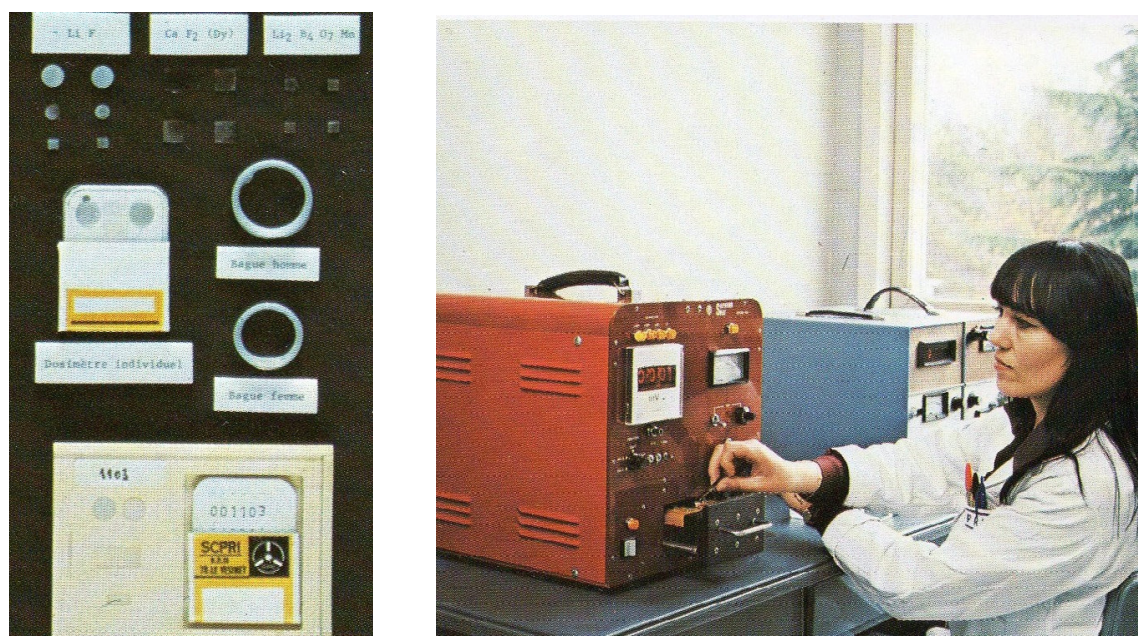

Figure 7.11 - Dosimètres TLD et lecture au SCPRI (SCPRI, 1979). 
Plus tard ces dosimètres ont été utilisés régulièrement pour la mesure du rayonnement ambiant naturel sur tout le territoire français à raison de 20 dosimètres par département (Biau et al., 1977).

Les demandes de tels dosimètres pour des études de poste ont été régulièrement adressées au SCPRI par des personnes compétentes en radioprotection (PCR).

Enfin, après 1994 à l'OPRI ces dosimètres ont été utilisés pour établir les premiers niveaux de référence diagnostique (NRD) par Hélène Beauvais-March et Marc Valero en collaboration avec des physiciens médicaux dans tout l'hexagone (Beauvais et al., 2003). Ces NRD ont fait l'objet, après des mesures complémentaires, d'un arrêté du 12 Février 2004 qui impose aux radiologues de contrôler l'exposition liée à leurs pratiques radiologiques. Le suivi de l'application se fait à l'IRSN (http://nrd.irsn.fr) avec Bernard Aubert (IRSN, 2010) responsable de l'Unité d'expertise en radioprotection médicale (UEM) de l'IRSN.

Par ailleurs pour les travailleurs exposés au niveau des mains, des bagues munies de dosimètres TLD pour mesurer les doses aux extrémités ont été régulièrement fournies dans le cadre d'abonnements en complément des dosimètres photographiques. Ainsi durant l'année 2000 environ 1800 dosimètres TLD étaient traités à l'OPRI (tableau II).

TABLEAU II

Bilan des doses en 2000 relatives aux mesures avec des dosimètres TLD. Doses aux extrémités (OPRI, 2001).

\begin{tabular}{lcccc}
\hline Type d'activité & $\begin{array}{c}\text { Etablissements } \\
\text { suivis }\end{array}$ & Personnes suivies & $\begin{array}{c}\text { Dose min } \\
\mathrm{mSv}\end{array}$ & $\begin{array}{c}\text { Dose max } \\
\mathrm{mSv}\end{array}$ \\
\hline Médecine nucléaire & 16 & 102 & 6 & 70 \\
Radiologie interventionnelle & 8 & 74 & 1,5 & 450 \\
Sources non scellées & 9 & 20 & 0.1 & 1 \\
Entreprises extérieures du & 4 & 17 & 0.2 & 4 \\
Nucléaire & 4 & 13 & 0.1 & 1 \\
Radiothérapie endocrinienne ${ }^{90} \mathrm{Sr}$ & 9 & 10 & 0.1 & 1 \\
Divers & 50 & 236 & & \\
Total & & & & \\
\hline
\end{tabular}

\subsubsection{Dosimétrie des neutrons}

Jusqu'en 2000, la technique utilisée pour la dosimétrie des neutrons rapides était le dosimètre photographique Kodak NTA. C'est une technique délicate et fastidieuse et ne permet pas d'obtenir les doses avec une grande précision. Délicate car, avant même l'envoi aux utilisateurs, les dosimètres doivent être placés durant une 
semaine dans un dispositif de séchage puis introduit dans une pochette thermosoudée pour éviter toute action de l'humidité. Ensuite la durée du développement photographique est bien plus grande que pour les dosimètres photographiques classiques, environ 20 minutes contre 5 minutes et les bains doivent être rigoureusement à température constante. De même le séchage des dosimètres après développement et fixage, doit être fait dans une enceinte particulièrement propre car, comme la lecture consiste à compter des traces au microscope, il ne faut risquer de prendre une poussière pour une trace laissée par un proton dans l'émulsion.

Fastidieuse car la lecture au microscope est très délicate pour des personnels qui ne sont pas des biologistes et dont ce n'est pas l'outil de travail habituel. En outre il faut compter les traces par unité de surface et souvent la répartition des traces n'est pas homogène (figure 7.12). En tout il faut compter une vingtaine de minutes contre moins d'une minute pour un dosimètre $\gamma, X$.

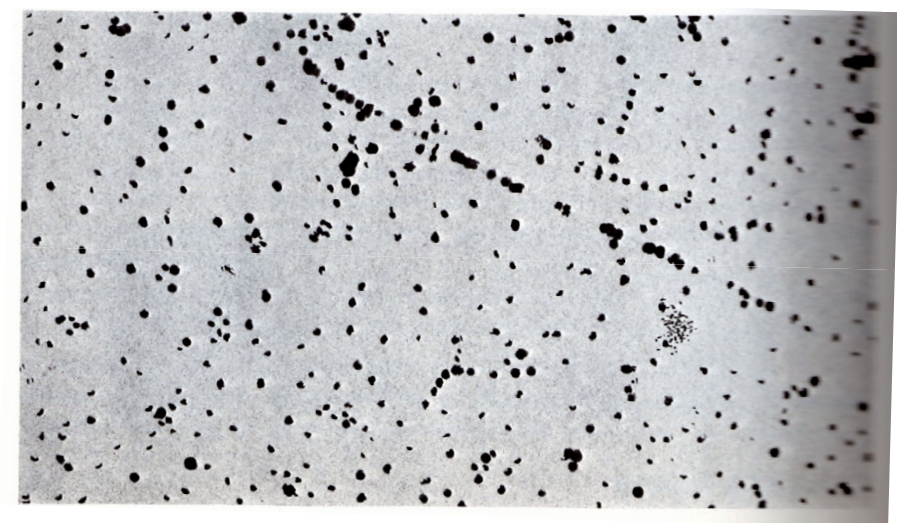

Figure 7.12 - Répartition de traces dans une émulsion nucléaire (Avan et al., 1973).

Le laboratoire ne traitait chaque mois qu' environ 500 dosimètres de ce type, car les personnels ayant une probabilité d'exposition aux neutrons rapides se trouvent essentiellement dans des installations de recherche du CEA ou du CNRS, dans des Installations nucléaires de base (INB) ou à la Défense qui disposent de leurs propres laboratoires de dosimétrie individuelle. Les 500 dosimètres étaient destinés surtout à des utilisateurs de sources scellées émettrices de neutrons comme l'AmériciumBéryllium ou du Californium 252, les premières dans des appareils de mesure d'humidité dans la recherche ou les travaux publics, les deuxièmes sous forme de grains pour la curiethérapie intra-cavitaire mais cette technique expérimentale mise en œuvre dans les années 1970, notamment au Centre Antoine Lacassagne de Nice a été vite abandonnée faute de résultat probant et en raison des difficultés de radioprotection. 
En 1998, l'OPRI a fait l'acquisition d'un système automatisé de la lecture de dosimètres constitués de détecteurs solides de traces PN3 qui a été mis en œuvre par l'équipe dirigée par Marc Valero avec Emmanuelle Girard et Éric Cale en 2000 (figures 7.13 et 7.14).

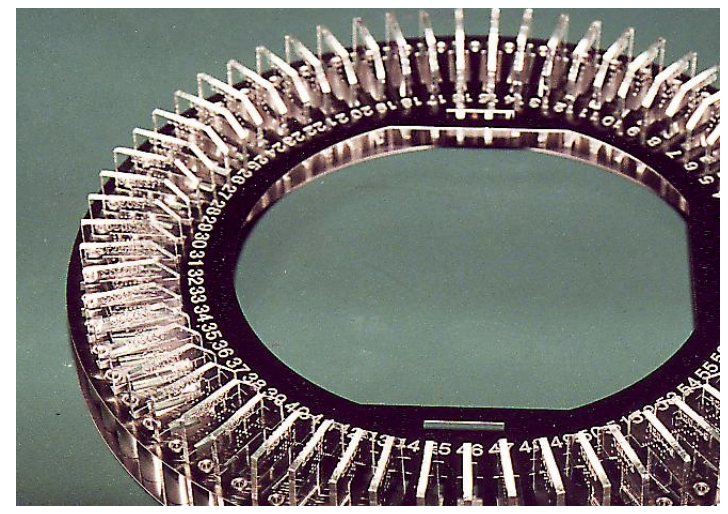

Figure 7.13 - Carrousel comportant les dosimètres PN3 pour lecture.

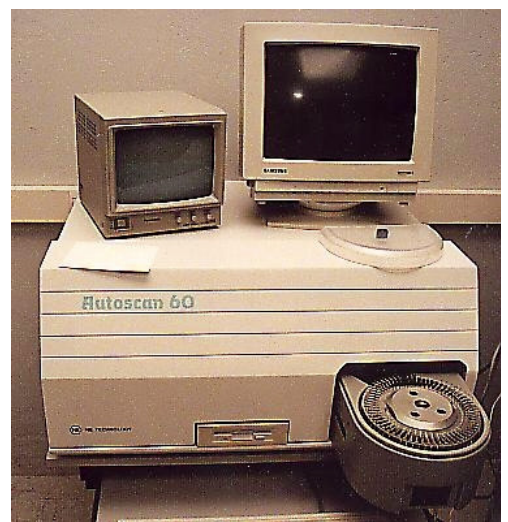

Figure 7.14 - Lecteur automatique de dosimètres PN3.

\subsubsection{Cas particulier de la dosimétrie des neutrons thermiques}

La mesure des neutrons thermiques se faisait à partir d'un dosimètre $\gamma, X$ classique mais doté d'un étui porte filtres particulier avec sur l'écran de cuivre l'ajout d'un carré de cadmium. Les neutrons thermiques étant capturés par le cadmium selon la réaction $(n, \gamma)$ les photons ainsi créés entrainent un noircissement sous forme de carré sur le dosimètre photographique et la densité mesurée dans cette zone permet d'évaluer la dose due aux neutrons thermiques (figure 7.15). 


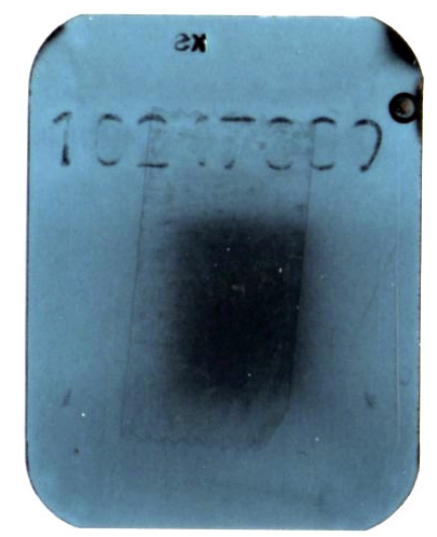

Figure 7.15 - Dosimètre photographique exposé à des neutrons thermiques.

Ce dosimètre a été exposé en laboratoire et présente une zone très nette correspondant à l'exposition aux neutrons thermiques, en pratique les neutrons thermiques sont accompagnés le plus souvent de rayonnements $\gamma$ qui impressionnent également le film et le contraste est moins évident.

\subsubsection{Cas particulier de la dosimétrie bêta}

Le dosimètre de base était particulièrement adapté à la mesure des rayonnements $\mathrm{X}$ et $\boldsymbol{\gamma}$ mais pour le rayonnement $\beta$ il ne disposait pas de plage de mesure spéciale sous $300 \mathrm{mg} / \mathrm{cm}^{2}$ comme dans certains dosimètres plus élaborés en milieu nucléaire notamment. Mais il y avait très peu d'abonné au SCPRI (OPRI) soumis à un risque d'exposition aux rayonnements $\beta$, sauf quelques utilisateurs de sources de Phosphore 32 dont le rayonnement très énergétique pouvait se mesurer correctement sur la plage nue comme pour les rayons $\mathrm{X}$.

Pour les rayonnements $\beta$ ou les électrons issus d'accélérateurs le dosimètre photographique le dosimètre photographique pouvait, sinon mesurer une dose au sens strict du terme, révéler des circonstances exceptionnelles d'exposition comme ce fut le cas lors de l'accident de Forbach qui a été élucidé grâce à l'exploitation en urgence des dosimètres photographiques (LCIE) des trois agents concernés (voir encadré L'accident de Forbach). 


\section{L'accident de Forbach}

Le 23 août 1991, le SCPRI est alerté par le médecin du travail d'une entreprise d'irradiation de matériaux par électrons de haute énergie située à Forbach. Lors d'une visite médicale de contrôle le médecin a constaté chez trois agents intérimaires des rougeurs cutanées inexplicables. Compte tenu de la potentialité d'exposition importante dans ce type d'installation et, malgré les dénégations du responsable de l'entreprise qui affirmait qu'une exposition était impossible, le SCPRI demande que les dosimètres soient transmis immédiatement au laboratoire (LCIE) pour lecture en urgence. Ces dosimètres ont été amenés le soir même par porteur au LCIE. À peine développés ces dosimètres interpellent Guy Leroy, responsable du laboratoire (voir chapitre 10), qui trouve leur aspect tout à fait anormal en raison, de toute évidence, de fortes doses d'électrons. Il informe immédiatement le SCPRI qui transmet l'information au médecin du travail et deux de ces agents sont hospitalisés en région parisienne. De fortes radiodermites ont été constatées, les victimes ont été soignées à l'hôpital militaire Percy de Clamart et l'employeur a fait l'objet de poursuites judiciaires en raison de manquements graves à la réglementation du travail.

\section{RÉFÉRENCES}

Amiel M., Clermont A., Jocteur-Monrosier D., Moroni J.P., Brun P. (1976) Étude dosimétrique au cours de l'angiographie cardiaque chez le jeune enfant, Annales de Radiologie 19, 623-628.

Avan L., Avan M., Blanc D., Tessier J.L. (1973) Ionographie - Émulsions - Détecteurs solides de traces. DOIN Editeurs, Paris.

Beauvais March H., Valero M., Biau A., Bourguignon M. (2003) Niveaux de référence diagnostique : spécificités de la demande française en radiologie, Radioprotection 38, $\mathrm{n}^{\circ} 2$.

Biau A., Moroni J.P. (1977) A review of existing information on external radiation from natural radioactivity in Europe, Rapport EURATOM.

IRSN (2010) Institut de radioprotection et de sûreté nucléaire, NRD Rapport DRPH/2010 - 1.

OPRI (2001) Rapport annuel 2000 d'activité de l'Office de Protection contre les Rayonnements Ionisants.

SCPRI (1979) Le Service Central de Protection contre les Rayonnements Ionisants, INSERM, Plaquette de présentation. 